\title{
Fish assemblages in a coastal bay adjacent to a network of marine protected areas in southern Brazil
}

\author{
André Pereira Cattani ${ }^{1 *}$, Fábio Gonçalves Daura Jorge ${ }^{2}$, Gisela Costa Ribeiro ${ }^{1}$, Leonardo Liberali \\ Wedekin ${ }^{3}$, Paulo César de Azevedo Simões Lopes ${ }^{4}$, Gabriel Martín Rupil ${ }^{1}$, Henry Louis Spach ${ }^{1}$ \\ ${ }^{1}$ Centro de Estudos do Mar, Universidade Federal do Paraná \\ (Av. Beira Mar S/N Caixa Postal 50002, 83255-000, Pontal do Paraná, Paraná, Brasil) \\ ${ }^{2}$ Universidade do Estado de Santa Catarina \\ (Avenida Madre Benvenuta, 2007 - Itacorubi, Florianópolis, SC, 88035-001) \\ ${ }^{3}$ Socioambiental Consultores Associados, Meio Ambiente \\ ${ }^{4}$ Universidade Federal de Santa Catarina \\ (R. Eng. Agronômico Andrei Cristian Ferreira, s/n - Trindade, Florianópolis, SC, 88040-900) \\ *Corresponding author: cattani.andre@gmail.com
}

\section{ABSTRACT}

Baía Norte (North Bay) in Santa Catarina State is considered a typical coastal bay and is surrounded by a network of Marine Protected Areas. The objectives of this study were to describe the composition of the demersal fish assemblage, identify seasonal and spatial structures on a fine scale and evaluate the role of habitat descriptors and abiotic variables affecting the fish assemblage structure. Seasonal samplings were conducted in 2005, using bottom trawls in six pre-established areas in Baía Norte in summer, fall, winter and spring. Simultaneously with each trawl, environmental data were collected with a multiparameter probe. Temporal and spatial differences in fish abundance were tested by a PERMANOVA. To illustratethe differences detected graphically we ran a canonical analysis of principal coordinates (CAP). The influence of environmental variables on the fish fauna was evaluated using a Distant Based Linear Model (DistLM) with Akaike's information criterion (AIC). A total of 9,888 specimens, distributed in 27 families and 62 species, were collected. Citharichthys spilopterus was the most abundant species. PERMANOVA detected differences for abundance between seasons, areas and interaction among all the factors. The DISTLM selected temperature and $\mathrm{pH}$. The results highlight seasonality as an important factor in the structuring of fish fauna of the study place.

Descriptors: Norte Bay, Ichthyofauna, Santa Catarina Island.

\section{Resumo}

A Baía Norte em Santa Catarina, é considerada como uma típica baía costeira, rodeada por uma rede de Áreas Marinhas Protegidas. Este estudo teve como objetivos descrever a composição e estrutura da comunidade de peixes, testar a influência dos padrões espaciais e sazonais e avaliar a influência das variáveis abióticas nos padrões de abundância. Amostragens sazonais foram realizadas por meio de arrasto de fundo em seis áreas predefinidas da Baía Norte no verão, outono, inverno e primavera no ano de 2005. Simultaneamente, foram coletados dados do ambiente com uma sonda multiparâmetro. Diferenças temporais e espaciais na abundância de peixes foram testados por uma análise PERMANOVA. Para visualizar graficamente as diferenças encontradas na PERMANOVA, foi realizada a análise canônica de coordenadas principais (CAP). Para avaliar a influência de variáveis ambientais sobre a fauna de peixes, foi aplicado um DISTLM usando critério de informação de Akaike (AIC). Foram coletados 9.888 espécimes, distribuídos em 27 famílias e 62 espécies. Citharichthys spilopterus foi a espécie dominante. A PERMANOVA detectou diferenças na abundância entre estações, áreas e interação entre todos os fatores. O DISTLM selecionou a temperatura e $\mathrm{pH}$. Os resultados enfatizam a sazonalidade como um fator importante para a estruturação da fauna de peixes do local de estudo.

Descritores: Baía Norte, Ictiofauna, Ilha de Santa Catarina. 


\section{INTRODUCTION}

Fish distribution patterns are shaped on different time scales that range from circadian or nictemeral cycles to interannual variations (MACI; BASSET, 2009), and interfere with ecological processes such as recruitment and reproduction (GARCIA et al., 2001; GREENWOOD; HILL, 2003; JAUREGUIZAR et al., 2004). In spatial terms, fish assemblage composition is organized on hierarchical scales, varying from meters to thousands of kilometers. On a regional scale (hundreds to thousands of kilometers), different factors structure an assemblage and these include the latitudinal temperature gradient, width and length of the estuary, vegetation cover (swamps and marshes), tidal range, rainfall and distance between estuaries and interfere with larval dispersal and species recruitment processes (BLABER, 2000; HARRISON; WHITIFIELD, 2006; SHEAVES; JOHNSTON, 2009; VILAR et al., 2013). On a local scale, salinity, dissolved oxygen and transparency are key factors affecting the occurrence of assemblages (BARLETTA et al., 2005; VILAR et al., 2011; 2013).

Human influence also alters spatio-temporal distribution patterns of fish assemblages. In estuarine and lagoon habitats, fish assemblages are potentially impacted by many anthropogenic activities with direct influence on food resources, distribution, diversity, reproduction, abundance, growth, survival and behavior of both resident and migratory species (MCDOWALL, 1988; ELLIOT; QUINTINO, 2007; BREHMER et al., 2013). Estuaries and coastal ecosystems are areas susceptible to the waste dumping and sewage discharge of a growing human population in the coastal zone (KENNISH, 1990), and are, further, still suffering from the effects of overfishing and tourism, as well as urbanization, agriculture, aquaculture and industrial development (RAZ-GUZMA; HUIDOBRO, 2002). Because of their position in the catchment area, these environments are among the most impacted by human activities (CABRAL et al., 2001; LOBRY et al., 2003).

Studies of the composition and structure of fish assemblages and the spatial and temporal variations in estuarine and coastal environments on the Brazilian coast are focused mainly in subtropical and temperate areas (BARLETTA et al., 2005). A recent comparative study of the fish assemblages in five estuaries along the Brazilian coast (Curuçá, Santa Cruz, Piraquê-Açú, Paranaguá and Lagoa dos Patos) found higher variability between estuaries than between the samples within each estuary, and showed that the composition of the assemblages is structured by both environmental factors (physical, hydrological and geographical characteristics of estuaries) and biological factors (larval recruitment and dispersal ability of species) (VILAR et al., 2013).

In southern Brazil, studies on fish assemblages in coastal environments, particularly estuaries, are more numerous in relation to the coast of the states of Rio Grande do Sul (GARCIA; VIEIRA, 2001a; GARCIA et al., 2001b; RAMOS; VIEIRA, 2001; GARCIA et al., 2003; BURNS et al., 2006) and Paraná (SPACH et al., 2004; OTERO et al., 2006; FALCÃO et al., 2006; SPACH et al., 2006; 2007; CONTENTE et al., 2011). The state of Santa Catarina has a strong fishing vocation but studies on fish assemblages on its coast are less common (MONTEIRO-NETO et al., 1990; RIBEIRO et al., 1997; 1999; BARREIRO et al., 2009, VILAR et al., 2011; RIBEIRO et al., 2014). This study was, therefore, designed to fill the current information gap, as well as to contribute to the understanding of ecological processes involving the fish assemblage in the Baia Norte, a coastal bay adjacent to a network of Marine Protected Areas on the central coast of Santa Catarina State. The main objectives were to: (1) describe the composition of the demersal fish assemblage; (2) identify seasonal and spatial structures on a fine scale; and (3) evaluate the role of habitat descriptors and abiotic variables affecting the fish assemblage structure. Ultimately, this information may provide support for future management plans and/or actions for the adjacent MPAs in the area; and increase ecological knowledge of fish assemblages on tropical and subtropical coasts.

\section{MATERIAL AND METHODS}

\section{STUDY AREA}

The North Bay (Baia Norte) of the island of Santa Catarina (2730' S; 48 $35^{\prime}$ W), southern Brazil (Fig. 1), is considered a typical coastal bay (SORIANO-SIERRA; SIERRA DE LEDO, 1998). It is a protected bay delimited by a mosaic of rocky shores, sandy beaches and mangrove areas. Its oceanographic parameters are similar to those of the adjacent open waters (SIMONASSI et al., 2010). Tidal and drift currents as well as the inflow of freshwater exercise considerable influence on the dynamics of this ecosystem. The bay has a surface area of approximately $200 \mathrm{~km}^{2}$, average depth of $5 \mathrm{~m}$ and a maximum depth of $21 \mathrm{~m}$. Water temperature varies markedly between seasons $\left(15-29^{\circ} \mathrm{C}\right)$. Most of the substrate in the inner bay 
is silt and mud. The tidal range is of approximately $1 \mathrm{~m}$ (SORIANO-SIERRA, 1999).

Our study area is surrounded by three Marine Protected Areas with different degrees of restriction: the Arvoredo Marine Biological Reserve (Arvoredo Reserve), the Anhatomirim Environmental Protection Area (Anhatomirim EPA) and the Carijós Ecological Station (Carijós Station). The Arvoredo Reserve is a no-take reserve created in 1990 (Decree 99142), located in adjacent coastal waters. The Carijós Station (Decree 94656), created in 1987, is also very restrictive to any resource use and visitation, and covers mainly mangrove and estuarine habitats within Baia Norte. The Anhatomirim EPA was created in 1992 (Decree 528) with the main purpose of protecting a resident Guiana dolphin population in North Bay (padronizar North Bay or Baia Norte!). It is a multiple-use protected area, which means that some resource use is permitted inside the MPA, but under specific regulations.

\section{SAMPLING}

Seasonal samplings were conducted in 2005. Using a 6-meter boat, bottom trawls were performed for 30 minutes in six pre-established areas in the North Bay (Figure 1) in the four seasons. The central month of each season was chosen as the sampling period, with samples taken on four non-consecutive days (at weekly intervals). Six trawls were performed on each day. Thus, 96 samples were obtained (6 trawls X 4 days X 4 months). Simultaneously with each trawl, salinity, temperature and $\mathrm{pH}$ were measured by means of a multiparameter probe. Rainfall data were provided by the Empresa de Pesquisa
Agropecuária e Extensão Rural de Santa Catarina (EPAGRI). Trawl net mesh sizes were $2.5 \mathrm{~cm}$ between opposite knots at the codend and $4 \mathrm{~cm}$ at the mouth, width of $8.65 \mathrm{~m}$ and height of 1.80 meters ( $15.57 \mathrm{~m}^{2}$ opening).

\section{STATISTICAL ANALYSIS}

Sampling months were grouped into four seasons: summer (December, January and February); fall (March, April and May); winter (June, July and August); and spring (September, October and November).

For data analysis, the analytical model used was complex: $X=\mu+\operatorname{Sea}+\operatorname{Sec}+\operatorname{Are}(\mathrm{Sec})+\operatorname{Sea} *$ Are $(\mathrm{Sec})$ $+e$, where $X=$ dependent variable; $\mu=$ mean; $S e a=$ season, $S e c=$ Sector, Are $=$ area; $e=$ error or residue. This model was used to test the differences between environmental parameters and population parameters of fish between seasons (summer, winter, fall and spring), between areas $(\mathrm{A}, \mathrm{B}, \mathrm{C}, \mathrm{D}, \mathrm{E}$ and $\mathrm{F})$ and sectors (north $=$ areas A, B and $C$; south $=$ areas D, E and F). The factors season and sector were fixed and the factor area was random, nested within the factor sector.

Temporal and spatial differences in fish abundance were tested by a multivariate analysis of variance using permutations (PERMANOVA - Permutational multivariate analysis of variance) (ANDERSON et al., 2008). A pairwise PERMANOVA was applied to a posteriori comparisons between factors showing significant differences in PERMANOVA ( $p$-value $<0.05)$. For graphical illustration of the differences detected we ran a canonical analysis of principal coordinates (CAP), which generates graphic clusters through permutation (ANDERSON et al., 2008). Within the CAP analysis,
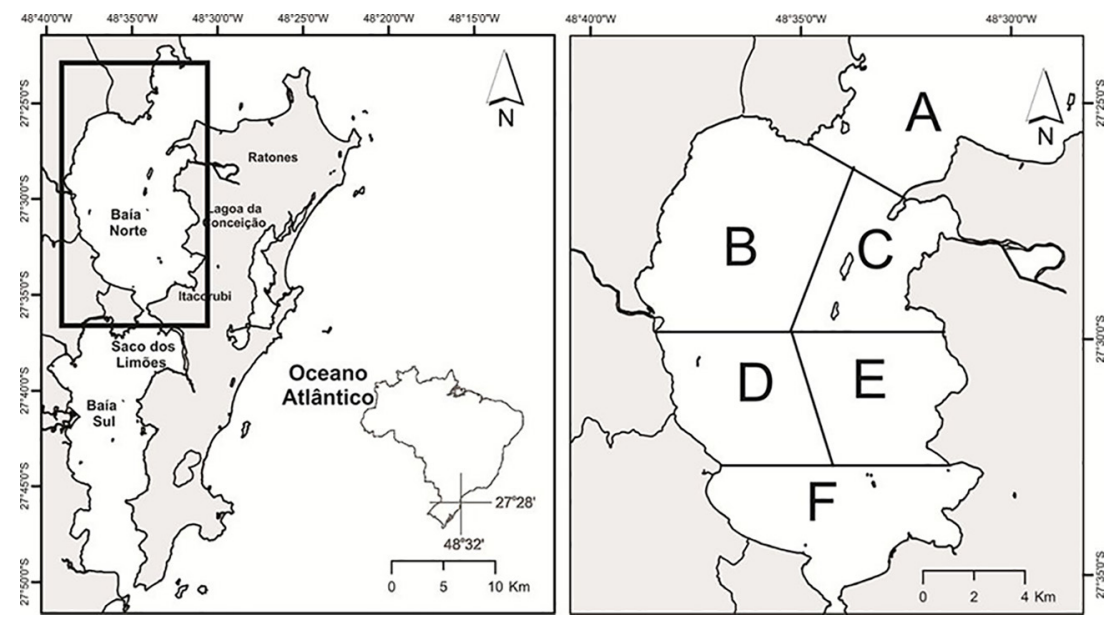

Figure 1. Map of the island of Santa Catarina showing the sampling areas in the North Bay. 
correlation Spearman at 0.4 was set to determine which species (vectors) were responsible for the clusters.

The differences in environmental parameters were also tested by a PERMANOVA. To evaluate the influence of environmental variables on the variability of fish data and to select the best explanatory model, we applied a DISTLM (Distance-Based Linear Model) using Akaike's information criterion (AIC) (ANDERSON et al., 2008). A stepwise procedure was adopted to test the importance of the independent variables. Environmental data were logtransformed $(\log (\mathrm{x}+1))$ and biotic data were square root transformed (CLARKE; WARWICK, 1994). Redundancy Analysis (dbRDA - distance-based redundancy analysis) was used to examine the influence of predictor variables on the spatial distribution of samples (ANDERSON et al., 2008). In all analyses, 9999 permutations were made. The similarity index used for abundance data was the BrayCurtis coefficient. The PERMANOVA for environmental variables was run on the basis of the similarity matrix obtained by Euclidean distance.

To assess the taxonomic differences between seasons in the area and determine the seasons with higher taxonomic complexity, we calculated the indices of Average Taxonomic Distinctness (Delta+ or AvTD) and Variation in Taxonomic Distinctness (Lambda+ or VarTD) with the presence/absence matrix (CLARKE; WARWICK, 1994).

To observe graphically whether the values of Average Taxonomic Distinctness and Variation in Taxonomic Distinctness of the seasons are within the expected taxonomic ranges, we constructed a funnel plot of AvTD and VarTD. We also prepared a biplot graphic display with both indices on each axis of the graph. A 95\% probability ellipse of species was superimposed on the biplot graphic display.

Taxonomic differences between the seasons were tested by univariate PERMANOVA, with the species richness, values of AvTD and VarTD as dependent variables and seasons as fixed factor.

\section{RESULTS}

\section{ENVIRONMENTAL VARIABLES}

The PERMANOVA run individually for each environmental variable (salinity, temperature, $\mathrm{pH}$ and rainfall) detected significant differences $(p<0.05)$ among seasons, with no differences among areas and sectors. In the paired comparison by pairwise PERMANOVA, in relation to salinity and $\mathrm{pH}$, except for the summer and spring, significant differences were detected between the other seasons (Table 1). For rainfall, there were no differences, except between summer and fall, and for temperature, there were differences between the four seasons.

Table 1. Pairwise PERMANOVA on the basis of the Euclidean distance of salinity, temperature and rainfall, $\log$ transformed $\log (\mathrm{X}+1)$ and $\mathrm{pH}$, comparing the seasons (summer, winter, fall and spring).

\begin{tabular}{lcccc}
\hline Seasons & $\begin{array}{l}\text { Salinity } \\
\mathrm{p}(\mathrm{perm})\end{array}$ & Temperature & $\mathrm{pH}$ & Rainfall \\
\hline $\begin{array}{l}\text { Summer, } \\
\text { Fall }\end{array}$ & $0.0341^{*}$ & $0.0025^{* *}$ & $0.0341^{*}$ & 0.6462 \\
$\begin{array}{l}\text { Summer, } \\
\text { Winter }\end{array}$ & $0.0078^{* *}$ & $0.0017^{* *}$ & $0.0078^{* *}$ & $0.0001^{* * *}$ \\
$\begin{array}{l}\text { Summer, } \\
\text { Spring }\end{array}$ & 0.8558 & $0.0023^{* *}$ & 0.8558 & $0.0001^{* * *}$ \\
$\begin{array}{l}\text { Fall, } \\
\text { Winter }\end{array}$ & $0.0061^{* *}$ & $0.0023^{* *}$ & $0.0061^{* *}$ & $0.0198^{*}$ \\
$\begin{array}{l}\text { Fall, } \\
\text { Spring }\end{array}$ & $0.0303^{*}$ & $0.0021^{* *}$ & $0.0303^{*}$ & $0.0137^{*}$ \\
$\begin{array}{l}\text { Winter, } \\
\text { Spring }\end{array}$ & $0.0106^{*}$ & $0.0021^{* *}$ & $0.0106^{*}$ & $0.0001^{* * *}$ \\
\hline$p<0.05^{*} ; p<0.01^{* * *} ; p<0.0001^{* * *}$ & & \\
\hline
\end{tabular}

Higher mean values of salinity were observed in the fall (mean $\pm \mathrm{SD}, 36.21 \pm 1.47$ ), followed by summer $(35.25 \pm 0.85)$, spring $(35.21 \pm 0.88)$ and winter $(32.67 \pm 3.24)$ (Figure 2a). In descending order, higher mean values of temperature were found in summer $(25.60 \pm 0.62)$, spring $(24.18 \pm 1.03)$, fall $(22.48 \pm 1.44)$ and winter (19.4 \pm 1.24 ) (Figure $2 \mathrm{~b}$ ). Regarding the $\mathrm{pH}$, higher mean values were registered in spring $(8.56 \pm$ $0.08)$, winter $(8.19 \pm 0.08)$, fall $(7.5 \pm 0.49)$ and summer $(7.21 \pm 0.64)$ (Figure 2c). Regading rainfall, higher mean values were verified in winter $(212.95 \pm 40.6 \mathrm{~mm})$, followed by fall $(174.8 \pm 84.04 \mathrm{~mm})$, summer $(126.3 \mathrm{~mm} \pm 1.77)$ and spring (86.2 $\mathrm{mm} \pm 5.92$ ) (Figure $2 \mathrm{~d}$ ).

\section{FISH ASSEMBLAGE}

A total of 9,888 specimens, distributed in 27 families and 62 species (Table 2), were collected in the study period. The richest family was Sciaenidae (13); followed by Tetraodontidae (6); Carangidae (5); Gerreidae (4); Achiridae, Clupeidae, Engraulidae and Paralichthyidae (3 each); and Ariidae, Haemulidae and Mugilidae (2 each) (Table 2). The other families had only a single species. The families with the highest catches (the ten most abundant families) were Paralichthyidae (3,296 individuals), 

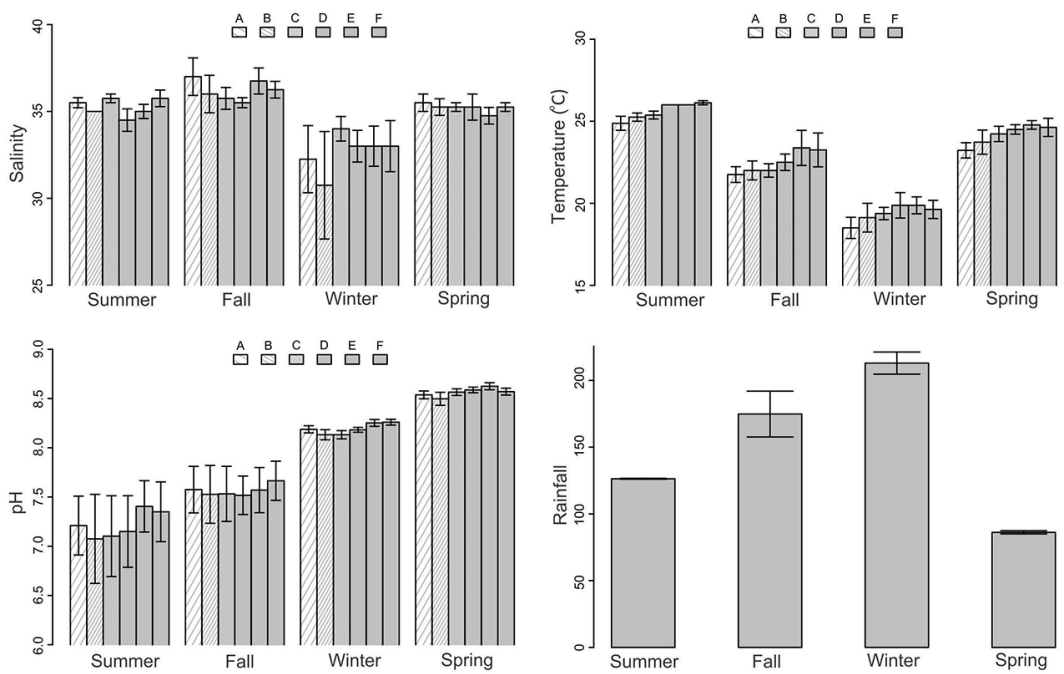

Figure 2. Mean values (standard errors on the bars) for salinity, temperature, $\mathrm{pH}$ and rainfall comparing the seasons and the six collection areas (A, $\mathrm{B}, \mathrm{C}, \mathrm{D}, \mathrm{E}$ and F) in the North Bay

Sciaenidae (1,820), Carangidae (992), Ariidae (904), Engraulidae (748), Cynoglossidae (639), Achiridae (254), Triglidae (252), Gerreidae (235) and Pristigasteridae (191). As for the weight, the greatest catches (ten dominant families) were found for Paralichthyidae $(63,865.2 \mathrm{~g})$, Ariidae (60,739.2 g), Sciaenidae (33,238.9 g), Engraulidae (17,887.5 g), Cynoglossidae (13,476.3 g), Trichiuridae $(8,526.24 \mathrm{~g})$, Gerreidae $(8,096.39 \mathrm{~g})$, Achiridae $(7,951.75$ g), Carangidae (7,677.71 g) and Triglidae $(5,481.44 \mathrm{~g})$.

In descending order, Citharichthys spilopterus, Genidens genidens, Stellifer rastrifer, Cetengraulis edentulus, Symphurus tessellatus, Chloroscombrus chrysurus, Micropogonias furnieri, Etropus crossotus, Selene setapinnis and Ctenosciaena gracilicirrhus were dominant in number of individuals, representing $80 \%$ of the total catch (abundance), while the catch of $C$. spilopterus alone corresponded to approximately $30 \%$ of the total. Anchoa spinifer, Anchovia clupeoides, Macrodon ancylodon, Mugil liza, Ophichthus gomesii, Paralichthys brasiliensis, Platanichthys platana, Trachinotus carolinus and Trinectes paulistanus were caught only once (Table 2).

The total catch in weight was $254,075.78 \mathrm{~g}$ (Table 2). G. genidens, C. spilopterus, C. edentulus, S. tessellatus, M. furnieri, S. rastrifer and E. crossotus corresponded, in descending order, to approximately $70 \%$ of the total weight. G. genidens and C. spilopterus amounted to $45 \%$ of the total catch in weight.

A total of 29 species were caught in all the seasons and 12 species in only one of the seasons. There was a greater richness in the fall ( 50 species), followed by the spring (45 species), winter (43 species) and summer (41 species) (Table 2).

Regarding areas, 25 species occurred in the six areas and 11 species presented exclusive occurrence in a single area. The highest species richness occurred in area $\mathrm{C}(44$ species), followed by areas F (42), E (41), A (40) and B and D (38 species each). Six species occurred exclusively in area $\mathrm{A}$ and only two species in area F. In the other areas, there was only one exclusive species (Table 2)

PERMANOVA detected significant differences $(p<0.05)$ for total abundance among seasons, areas and interaction between all factors (seasons, areas and sector), with the higher abundance mean values in areas $\mathrm{C}$ and $\mathrm{E}$ in the fall and lower menans in area $\mathrm{A}$ in the winter and area $\mathrm{D}$ in the summer (Table 3 and Figure 3). In paired comparisons (pairwise PERMANOVA) within each season, major differences between areas were observed in the spring, followed by winter, fall and summer (Table 4).

Differences in mean values of abundance among seasons by PERMANOVA as well as species that have contributed to such differences can be observed graphically in the canonical analysis of principal coordinates (CAP). The canonical correlation of the two axes obtained by the analysis was $\delta_{1}=0.8052$ and $\delta_{2}=0.7909$. There was a clear separation, along the first axis, between the summer and winter samples and, along axis 2, between the spring and fall (Figure 4). The high abundance of C. chrysurus, E crossotus and $S$. tyleri in the winter and fall samples, $S$. setapinnis and C. microlepidotus in the fall and summer, M. furnieri in the summer and C. edentulus in the spring, 
Table 2. List of species, number of individuals (n), weight (W), mean, minimum and maximum total length (TL), season and areas (higher abundances to the left) of fish caught in the North Bay.

\begin{tabular}{|c|c|c|c|c|c|c|}
\hline Famíly/Species & $\mathrm{n}$ & $\mathrm{W}(\mathrm{g})$ & $\begin{array}{c}\text { Mean } \\
\text { TL(mm) }\end{array}$ & $\begin{array}{c}\text { Mín-Máx } \\
\text { TL(mm) }\end{array}$ & Season & Area \\
\hline \multicolumn{7}{|l|}{ Achiridae } \\
\hline Achirus declivis & 16 & 1273.06 & 143.44 & $78-192$ & $\mathrm{su}>\mathrm{sp}=\mathrm{fa}=\mathrm{wi}$ & $\mathrm{C}>\mathrm{E}>\mathrm{D}$ \\
\hline Achirus lineatus & 237 & 6623 & 104 & $64-186$ & $\mathrm{sp}>\mathrm{su}>\mathrm{fa}>\mathrm{wi}$ & $\mathrm{F}>\mathrm{C}>\mathrm{E}>\mathrm{D}>\mathrm{B}>\mathrm{A}$ \\
\hline Trinectes paulistanus & 1 & 55.69 & 135 & 135 & $\mathrm{fa}$ & $\mathrm{A}$ \\
\hline \multicolumn{7}{|l|}{ Ariidae } \\
\hline Genidens barbus & 6 & 495.22 & 207.5 & $177-285$ & $w i>s p$ & $\mathrm{~B}>\mathrm{F}>\mathrm{C}$ \\
\hline Genidens genidens & 898 & 60244.10 & 193.62 & $55-320$ & $\mathrm{sp}>\mathrm{su}>\mathrm{wi}>\mathrm{fa}$ & $\mathrm{E}>\mathrm{C}>\mathrm{B}>\mathrm{D}>\mathrm{F}>\mathrm{A}$ \\
\hline \multicolumn{7}{|l|}{ Batrachoididae } \\
\hline Porichthys porosissimus & 5 & 307.52 & 166.6 & $39-215$ & $\mathrm{wi}>\mathrm{fa}=\mathrm{sp}=\mathrm{su}$ & $\mathrm{A}>\mathrm{B}=\mathrm{C}$ \\
\hline \multicolumn{7}{|l|}{ Carangidae } \\
\hline Chloroscombrus chrysurus & 575 & 4487.91 & 86.81 & $21-190$ & wi $>$ fa $>$ su $>$ sp & $\mathrm{B}>\mathrm{E}>\mathrm{F}>\mathrm{A}>\mathrm{C}>\mathrm{D}$ \\
\hline Oligoplites saurus & 6 & 58.09 & 114.67 & $107-126$ & wi $>$ sp & $\mathrm{B}>\mathrm{E}>\mathrm{D}$ \\
\hline Selene setapinnis & 294 & 2006.67 & 78.38 & $46-180$ & $\mathrm{su}>\mathrm{fa}>\mathrm{wi}>\mathrm{sp}$ & $\mathrm{B}>\mathrm{E}=\mathrm{F}>\mathrm{A}>\mathrm{C}=\mathrm{D}$ \\
\hline Selene vomer & 116 & 1089.27 & 65.94 & $31-209$ & $\mathrm{su}>\mathrm{fa}>\mathrm{sp}>\mathrm{wi}$ & $\mathrm{F}>\mathrm{D}>\mathrm{B}>\mathrm{E}>\mathrm{A}$ \\
\hline Trachinotus carolinus & 1 & 35.77 & 141 & 141 & $\mathrm{fa}$ & $\mathrm{F}$ \\
\hline \multicolumn{7}{|l|}{ Centropomidae } \\
\hline Centropomus parallelus & 5 & 551.04 & 226.2 & $223-232$ & $w i=f a>s p$ & $\mathrm{C}=\mathrm{F}>\mathrm{E}$ \\
\hline \multicolumn{7}{|l|}{ Clupeidae } \\
\hline Harengula clupeola & 18 & 594.29 & 140.39 & $90-176$ & wi $>f a=s p$ & $\mathrm{~B}>\mathrm{C}=\mathrm{D}=\mathrm{E}$ \\
\hline Opisthonema oglinum & 5 & 133.55 & 144.6 & $130-158$ & $\mathrm{wi}>\mathrm{fa}$ & $\mathrm{D}>\mathrm{B}$ \\
\hline Platanichthys platana & 1 & 1.61 & 54 & 54 & $\mathrm{sp}$ & \\
\hline \multicolumn{7}{|l|}{ Cynoglossidae } \\
\hline Symphurus tessellatus & 639 & 13476.31 & 140.77 & $90-195$ & $\mathrm{fa}>\mathrm{su}>\mathrm{wi}>\mathrm{sp}$ & $\mathrm{B}>\mathrm{C}>\mathrm{E}>\mathrm{F}>\mathrm{A}>\mathrm{D}$ \\
\hline \multicolumn{7}{|l|}{ Engraulidae } \\
\hline Anchoa spinifer & 1 & 21.28 & 150 & 150 & $\mathrm{sp}$ & A \\
\hline Anchovia clupeoides & 1 & 6.71 & 100 & 100 & $\mathrm{fa}$ & A \\
\hline Cetengraulis edentulus & 746 & 17859.49 & 132.05 & $88-174$ & $\mathrm{fa}>\mathrm{sp}>\mathrm{wi}>\mathrm{su}$ & $\mathrm{E}>\mathrm{D}>\mathrm{B}>\mathrm{F}>\mathrm{C}>\mathrm{A}$ \\
\hline \multicolumn{7}{|l|}{ Ephippidae } \\
\hline Chaetodipterus faber & 60 & 3927.86 & 112.42 & $28-397$ & $\mathrm{sp}>$ wi $>\mathrm{fa}>\mathrm{su}$ & $\mathrm{C}>\mathrm{B}>\mathrm{D}>\mathrm{F}>\mathrm{E}>\mathrm{A}$ \\
\hline \multicolumn{7}{|l|}{ Gerreidae } \\
\hline Diapterus rhombeus & 53 & 2326.97 & 138.43 & $105-176$ & $\mathrm{sp}>\mathrm{wi}>\mathrm{fa}$ & $\mathrm{C}>\mathrm{E}>\mathrm{F}>\mathrm{A}$ \\
\hline Eucinostomus argenteus & 70 & 1156.88 & 107.44 & $47-150$ & $\mathrm{fa}>$ wi $>$ su $>$ sp & $\mathrm{F}>\mathrm{C}>\mathrm{D}>\mathrm{E}>\mathrm{B}>\mathrm{A}$ \\
\hline Eucinostomus gula & 44 & 1145.16 & 122.84 & $89-148$ & wi $>$ su $>$ sp $>$ fa & $\mathrm{E}>\mathrm{D}>\mathrm{F}>\mathrm{A}>\mathrm{B}=\mathrm{C}$ \\
\hline Eucinostomus melanopterus & 68 & 3467.38 & 165.54 & $141-201$ & $w i=f a>s p$ & $\mathrm{~F}>\mathrm{C}>\mathrm{E}>\mathrm{D}$ \\
\hline \multicolumn{7}{|l|}{ Gobiidae } \\
\hline Gobionellus oceanicus & 8 & 213.44 & 193 & $160-221$ & $\mathrm{su}>\mathrm{fa}$ & $\mathrm{F}>\mathrm{B}=\mathrm{E}$ \\
\hline \multicolumn{7}{|l|}{ Haemulidae } \\
\hline Orthopristis ruber & 72 & 3156.8 & 132.75 & $64-259$ & $\mathrm{sp}>\mathrm{su}>\mathrm{wi}>\mathrm{fa}$ & $\mathrm{C}>\mathrm{F}>\mathrm{E}>\mathrm{A}$ \\
\hline Pomadasys corvinaeformis & 14 & 132.29 & 87.57 & $74-117$ & $\mathrm{fa}>$ wi & $\mathrm{C}>\mathrm{D}=\mathrm{F}$ \\
\hline \multicolumn{7}{|l|}{ Monacanthidae } \\
\hline Stephanolepis hispidus & 3 & 5.56 & 44.67 & $39-49$ & $\mathrm{su}=\mathrm{fa}=\mathrm{wi}$ & $\mathrm{C}=\mathrm{E}=\mathrm{F}$ \\
\hline \multicolumn{7}{|l|}{ Mugilidae } \\
\hline Mugil curema & 3 & 746.35 & 286 & $248-327$ & $\mathrm{su}>\mathrm{fa}$ & $\mathrm{D}>\mathrm{E}$ \\
\hline Mugil liza & 1 & 677.05 & 415 & 415 & $\mathrm{su}$ & A \\
\hline
\end{tabular}


Continued Table 2.

\begin{tabular}{|c|c|c|c|c|c|c|}
\hline Muraenidae & & & & & & \\
\hline Gymnothorax ocellatus & 3 & 625.61 & 489.33 & $480-498$ & $\mathrm{sp}>\mathrm{fa}$ & $\mathrm{B}>\mathrm{E}$ \\
\hline \multicolumn{7}{|l|}{ Ophichthidae } \\
\hline Ophichthus gomesii & 1 & 84.27 & 441 & 441 & $\mathrm{su}$ & $\mathrm{C}$ \\
\hline \multicolumn{7}{|l|}{ Paralichthyidae } \\
\hline Citharichthys spilopterus & 2955 & 55281.16 & 120.41 & $40-198$ & wi $>$ fa $>$ sp $>$ su & $\mathrm{E}>\mathrm{C}>\mathrm{B}>\mathrm{D}>\mathrm{F}>\mathrm{A}$ \\
\hline Etropus crossotus & 340 & 8583.99 & 94.32 & $55-132$ & $\mathrm{fa}>\mathrm{wi}>\mathrm{sp}>\mathrm{su}$ & $\mathrm{D}>\mathrm{C}>\mathrm{F}>\mathrm{B}>\mathrm{E}>\mathrm{A}$ \\
\hline Paralichthys brasiliensis & 1 & 0 & 482 & 482 & wi & \\
\hline \multicolumn{7}{|l|}{ Pristigasteridae } \\
\hline Pellona harroweri & 191 & 2814.9 & 101.86 & $57-175$ & $\mathrm{fa}>\mathrm{sp}>\mathrm{su}>$ wi & $\mathrm{B}>\mathrm{C}>\mathrm{A}>\mathrm{F}>\mathrm{D}>\mathrm{E}$ \\
\hline \multicolumn{7}{|l|}{ Rhinobatidae } \\
\hline Zapteryx brevirostris & 2 & 1073.4 & 412 & $360-464$ & $\mathrm{fa}=\mathrm{sp}$ & $\mathrm{A}=\mathrm{C}$ \\
\hline \multicolumn{7}{|l|}{ Sciaenidae } \\
\hline Ctenosciaena gracilicirrhus & 273 & 4153.93 & 100.97 & $62-138$ & $\mathrm{fa}>\mathrm{su}>\mathrm{sp}>\mathrm{wi}$ & $\mathrm{E}>\mathrm{C}>\mathrm{A}>\mathrm{F}>\mathrm{D}>\mathrm{B}$ \\
\hline Cynoscion jamaicensis & 42 & 520.23 & 99.52 & $60-180$ & $\mathrm{fa}>\mathrm{su}>$ wi & $\mathrm{C}>\mathrm{A}>\mathrm{B}>\mathrm{E}>\mathrm{D}>\mathrm{F}$ \\
\hline Cynoscion leiarchus & 5 & 117.24 & 131.2 & $113-147$ & $\mathrm{su}>\mathrm{fa}$ & $\mathrm{D}>\mathrm{E}$ \\
\hline Cynoscion microlepidotus & 115 & 997.06 & 87.89 & 41-199 & $\mathrm{su}>\mathrm{fa}$ & $\mathrm{E}>\mathrm{C}>\mathrm{B}>\mathrm{D}>\mathrm{A}>\mathrm{F}$ \\
\hline Isopisthus parvipinnis & 16 & 551.33 & 136.88 & $66-190$ & $\mathrm{fa}>\mathrm{su}>\mathrm{sp}>\mathrm{wi}$ & $\mathrm{A}>\mathrm{C}>\mathrm{B}=\mathrm{F}$ \\
\hline Macrodon ancylodon & 1 & 0.68 & 55 & 55 & & A \\
\hline Menticirrhus americanus & 56 & 5358.68 & 186.07 & $88-408$ & $\mathrm{su}>\mathrm{sp}=\mathrm{fa}=\mathrm{wi}$ & $\mathrm{F}>\mathrm{C}>\mathrm{B}>\mathrm{D}>\mathrm{E}>\mathrm{A}$ \\
\hline Micropogonias furnieri & 448 & 10278.52 & 118.53 & $55-223$ & $\mathrm{su}>\mathrm{sp}>\mathrm{fa}>\mathrm{wi}$ & $\mathrm{B}>\mathrm{C}>\mathrm{D}>\mathrm{E}>\mathrm{F}>\mathrm{A}$ \\
\hline Odontoscion dentex & 2 & 26.1 & 98.5 & $78-119$ & $\mathrm{su}$ & $\mathrm{B}$ \\
\hline Paralonchurus brasiliensis & 106 & 1322.85 & 111.89 & $45-194$ & $\mathrm{sp}>\mathrm{su}>\mathrm{wi}>\mathrm{fa}$ & $\mathrm{A}>\mathrm{B}>\mathrm{C}=\mathrm{D}$ \\
\hline Stellifer brasiliensis & 5 & 27.21 & 84.8 & $70-97$ & $\mathrm{su}=\mathrm{sp}>\mathrm{fa}$ & $A>B$ \\
\hline Stellifer rastrifer & 747 & 9858.69 & 99.08 & $33-178$ & $\mathrm{fa}>\mathrm{su}>\mathrm{sp}>$ wi & $\mathrm{C}>\mathrm{B}>\mathrm{F}>\mathrm{A}>\mathrm{E}>\mathrm{D}$ \\
\hline Umbrina coroides & 3 & 3.09 & 46.33 & $45-47$ & wi & A \\
\hline \multicolumn{7}{|l|}{ Serranidae } \\
\hline Diplectrum radiale & 31 & 2458.53 & 176.84 & $139-213$ & $\mathrm{wi}>\mathrm{fa}>\mathrm{sp}=\mathrm{su}$ & $\mathrm{E}>\mathrm{F}>\mathrm{C}=\mathrm{D}>\mathrm{A}$ \\
\hline \multicolumn{7}{|l|}{ Sparidae } \\
\hline Archosargus rhomboidalis & 21 & 2696.67 & 192.52 & $131-245$ & $\mathrm{su}>\mathrm{sp}=\mathrm{wi}>\mathrm{fa}$ & $\mathrm{E}>\mathrm{C}>\mathrm{F}$ \\
\hline \multicolumn{7}{|l|}{ Stromateidae } \\
\hline Peprilus paru & 41 & 1336.7 & 98.1 & $24-220$ & $\mathrm{fa}>\mathrm{sp}>\mathrm{su}>$ wi & $\mathrm{B}>\mathrm{A}>\mathrm{E}>\mathrm{D}>\mathrm{C}=\mathrm{F}$ \\
\hline \multicolumn{7}{|l|}{ Synodontidae } \\
\hline Synodus foetens & 21 & 1864.93 & 231.81 & 114-309 & $\mathrm{fa}>\mathrm{sp}>\mathrm{wi}>\mathrm{su}$ & $\mathrm{E}>\mathrm{C}>\mathrm{F}>\mathrm{D}$ \\
\hline \multicolumn{7}{|l|}{ Tetraodontidae } \\
\hline Lagocephalus laevigatus & 34 & 457.26 & 83.5 & $47-148$ & $\mathrm{sp}>\mathrm{su}>\mathrm{wi}$ & $\mathrm{B}>\mathrm{D}>\mathrm{A}=\mathrm{C}=\mathrm{F}>\mathrm{E}$ \\
\hline Sphoeroides sp. & 2 & 26.45 & 84 & $80-88$ & $\mathrm{sp}$ & $\mathrm{F}$ \\
\hline Sphoeroides greeleyi & 6 & 94.83 & 90.67 & $56-106$ & $\mathrm{sp}$ & $\mathrm{D}$ \\
\hline Sphoeroides spengleri & 34 & 210.88 & 64 & $28-113$ & $\mathrm{sp}>\mathrm{fa}>\mathrm{su}$ & $\mathrm{C}>\mathrm{E}>\mathrm{F}>\mathrm{A}=\mathrm{D}>\mathrm{B}$ \\
\hline Sphoeroides testudineus & 29 & 2704 & 137.38 & $28-242$ & $\mathrm{sp}>\mathrm{fa}>\mathrm{su}>\mathrm{wi}$ & $\mathrm{C}>\mathrm{D}>\mathrm{E}>\mathrm{F}>\mathrm{B}>\mathrm{A}$ \\
\hline Sphoeroides tyleri & 45 & 262.58 & 59.04 & $31-93$ & $w i>f a>s p$ & $\mathrm{D}>\mathrm{B}=\mathrm{E}>\mathrm{C}>\mathrm{A}=\mathrm{F}$ \\
\hline \multicolumn{7}{|l|}{ Trichiuridae } \\
\hline Trichiurus lepturus & 93 & 8526.24 & 388.4 & $67-1180$ & $\mathrm{su}>\mathrm{sp}>\mathrm{fa}>\mathrm{wi}$ & $\mathrm{C}>\mathrm{A}>\mathrm{F}>\mathrm{B}>\mathrm{E}>\mathrm{D}$ \\
\hline \multicolumn{7}{|l|}{ Triglidae } \\
\hline Prionotus punctatus & 252 & 5481.44 & 109,71 & $35-187$ & $\mathrm{su}>\mathrm{fa}>\mathrm{sp}>\mathrm{wi}$ & $\mathrm{E}>\mathrm{B}>\mathrm{F}>\mathrm{C}=\mathrm{D}>\mathrm{A}$ \\
\hline TOTAL & 9888 & 254075.78 & & & & \\
\hline
\end{tabular}


Table 3. PERMANOVA based on Bray-Curtis similarity for total abundance (transformed into square root) of fish caught in the North Bay. Factors: season $=$ Ses, sector $=\mathrm{Sec}$ and area $=$ Are $(\mathrm{Sec}) . \mathrm{df}=$ degrees of freedom; $\mathrm{MS}=$ sum of mean squares .

\begin{tabular}{lcccc}
\hline Source & df & MS & Pseudo-F & $p($ perm) \\
\hline Sea & 3 & 8501.4 & 3.5741 & 0.0001 \\
Sec & 1 & 10110 & 1.8125 & 0.303 \\
Are(Sec) & 4 & 5578.2 & 3.9197 & 0.0001 \\
Sea x Sec & 3 & 2914.2 & 1.2252 & 0.2164 \\
Sea $x$ Are(Sec) & 12 & 2378.6 & 1.6714 & 0.0001 \\
Res & 72 & 1423.1 & & \\
\hline
\end{tabular}

Table 4. Pairwise PERMANOVA comparing areas in each season based on Curtis similarity for total abundance (transformed into square root) of fish caught in the North Bay.

\begin{tabular}{lcccc}
\hline & Winter & Spring & Fall & Summer \\
Area & P(perm) & & & 0.0832 \\
\hline A, B & $0.0283^{*}$ & $0.0305^{*}$ & 0.8261 & 0.1478 \\
A, C & $0.0315^{*}$ & $0.0277^{*}$ & 0.082 & 0.4508 \\
A, D & $0.0275^{*}$ & $0.0296^{*}$ & $0.0252^{*}$ & 0.082 \\
A, E & $0.0302^{*}$ & $0.03^{*}$ & 0.0599 & 0.1661 \\
A, F & $0.0284^{*}$ & $0.029^{*}$ & 0.1188 & $0.0286^{*}$ \\
B, C & $0.0306^{*}$ & $0.027^{*}$ & 0.0853 & 0.0831 \\
B, D & $0.0285^{*}$ & $0.0292^{*}$ & $0.0286^{*}$ & 0.1142 \\
B, E & $0.0267^{*}$ & $0.0274^{*}$ & $0.0257^{*}$ & 0.374 \\
B, F & $0.0288^{*}$ & $0.028^{*}$ & $0.0269^{*}$ & 0.3432 \\
C, D & $0.0287^{*}$ & 0.0847 & 0.1143 & 0.1967 \\
C, E & 0.0597 & $0.0307^{*}$ & 0.0596 & 0.0876 \\
C, F & $0.0266^{*}$ & $0.0293^{*}$ & 0.2641 & 0.457 \\
D, E & 0.1685 & 0.0871 & 0.14 & 0.3632 \\
D, F & $0.0305^{*}$ & 0.2917 & 0.0573 & 0.7462 \\
E, F & 0.0615 & 0.2063 & 0.2596 &
\end{tabular}

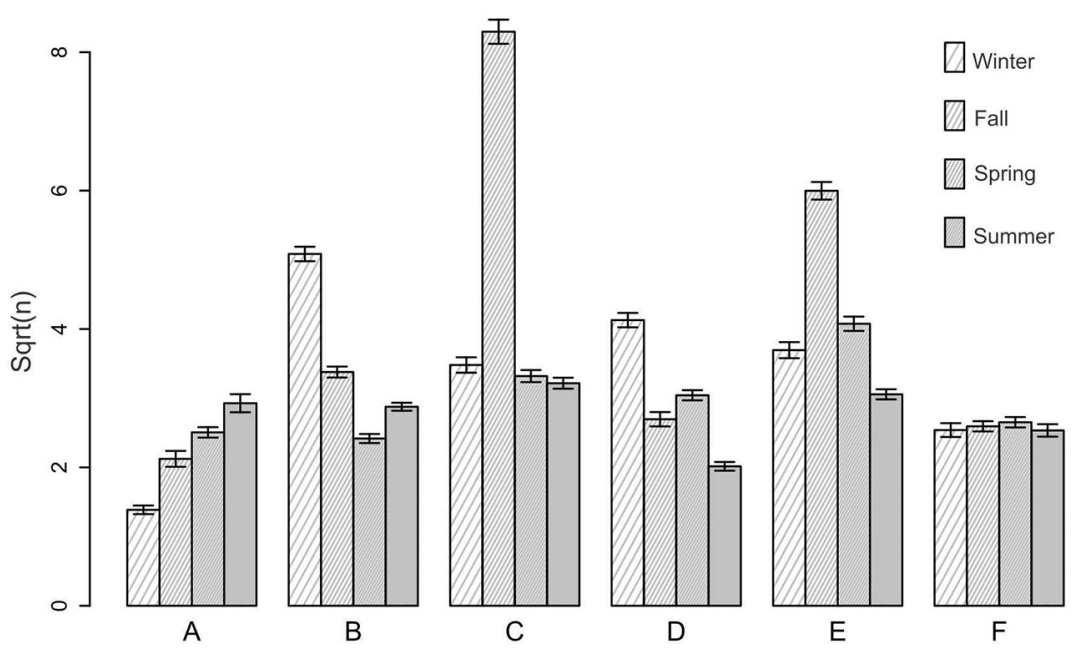

Figure 3. Mean values (standard error on the bars) of the square root of the abundance of fish caught in winter, fall, spring and summer in areas A, B, C, D, E, F of the North Bay. 
were responsible for such differences (Figure 4). In relation to the areas, the CAP plot demonstrated a clear separation of samples taken in areas A, B and $\mathrm{C}$ and a grouping of areas D, E and F (Figure 5). The high abundance of E. melanopterus and A. lineatus in these three areas $(\mathrm{D}, \mathrm{E}$ and $\mathrm{F}$ ) and of L. laevigatus in area B were responsible for the pattern observed (Figure 5). The canonical correlation of the two axes given by the analysis was de $\delta_{1}=0.8282$ and $\delta_{2}=0.6727$.

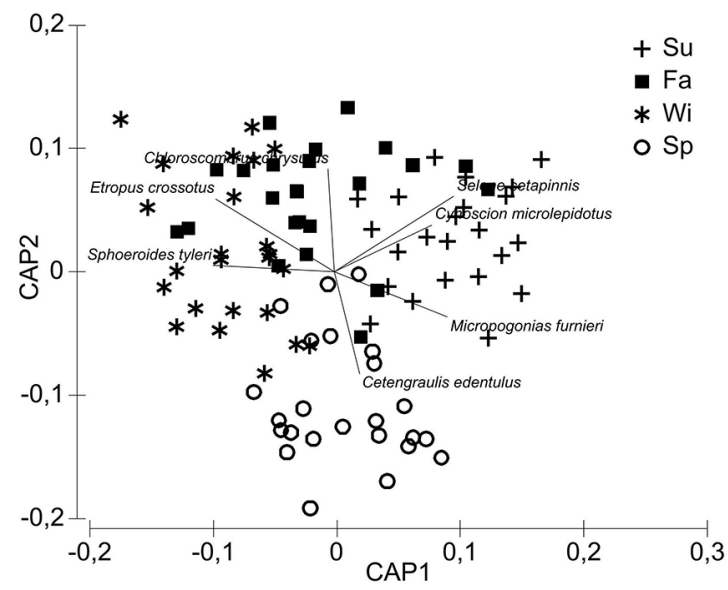

Figure 4. Results of the canonical analysis of principal coordinates (CAP) for the species of the North Bay that contributed to the differences between seasons (Summer $=\mathrm{Su}$; Fall $=\mathrm{Fa}$; Winter $=$ $\mathrm{Wi}$; Spring $=\mathrm{Sp}$ ). Vector of species based on Spearman correlation values $>0.5$.

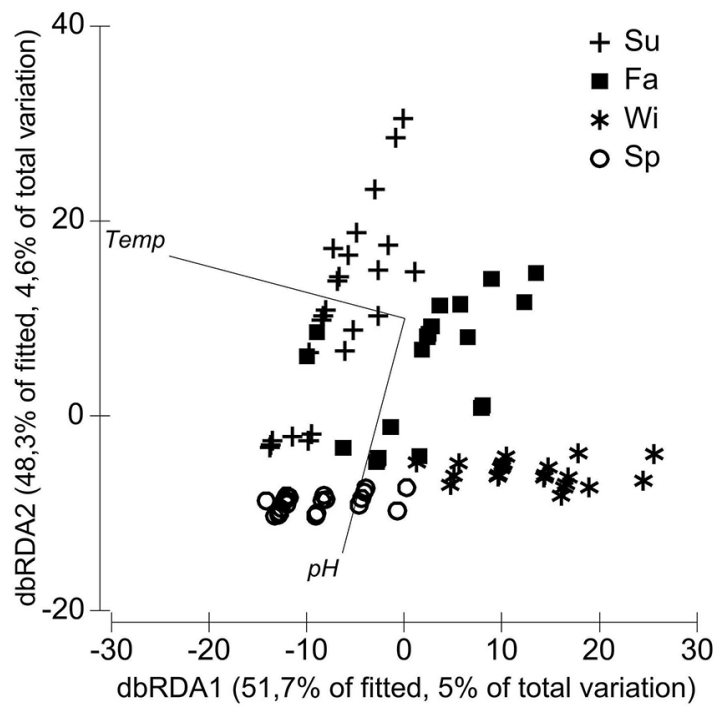

Figure 5. Result of the canonical analysis of principal coordinates (CAP) for the species of the North Bay that contributed to the differences between areas. Vector of species based on Spearman correlation values $>0.5(p>0.5)$.
The linear model selected by DISTLM included the temperature and $\mathrm{pH}$ as determinants for the variation of numerical abundance. Despite the significance of all the predictor variables in the model, the higher percentages of explanation were observed for the selected variables (Table 6). The graphical response of DISTLM provided by dbRDA pointed to a relationship of temperature with summer and fall samples (axis 1), and of $\mathrm{pH}$ with spring samples (axis 2; Figure 6).

Table 5. Result of the DISTLM analysis with $p$-value of the permutation and the percentage of explanation of variables for the selected model.

\begin{tabular}{lcc}
\hline Variable & $\mathrm{P}($ Perm $)$ & Proportion \\
\hline Rainfall & 0.0074 & $2.49 \mathrm{E}-02$ \\
Temperature & 0.0002 & $4.92 \mathrm{E}-02$ \\
$\mathrm{pH}$ & 0.0001 & $4.63 \mathrm{E}-02$ \\
Salinity & 0.0299 & $2.21 \mathrm{E}-02$ \\
\hline
\end{tabular}

Table 6. Result of univariate PERANOVA for richness, taxonomic distinctness (AvTD) and variation in taxonomic distinctness (VarTD) for the North Bay, with season as factor.

\begin{tabular}{lccccc}
\hline Source & & $\mathrm{df}$ & $\mathrm{MS}$ & Pseudo-F & $\mathrm{p}$ (perm) \\
\hline \multirow{2}{*}{ Richness } & Season & 3 & 106.4 & 0.73123 & \multirow{2}{*}{0.5686} \\
& Res & 92 & 145.29 & & \\
\hline \multirow{2}{*}{ AvTD } & Season & 3 & 0.64927 & 0.79938 & \multirow{2}{*}{0.7209} \\
& Res & 92 & 0.81222 & & \\
\multirow{2}{*}{ VarTD } & Season & 3 & 3.8144 & 0.5753 & \multirow{2}{*}{0.6506} \\
& Res & 92 & 6.6302 & & \\
\hline
\end{tabular}

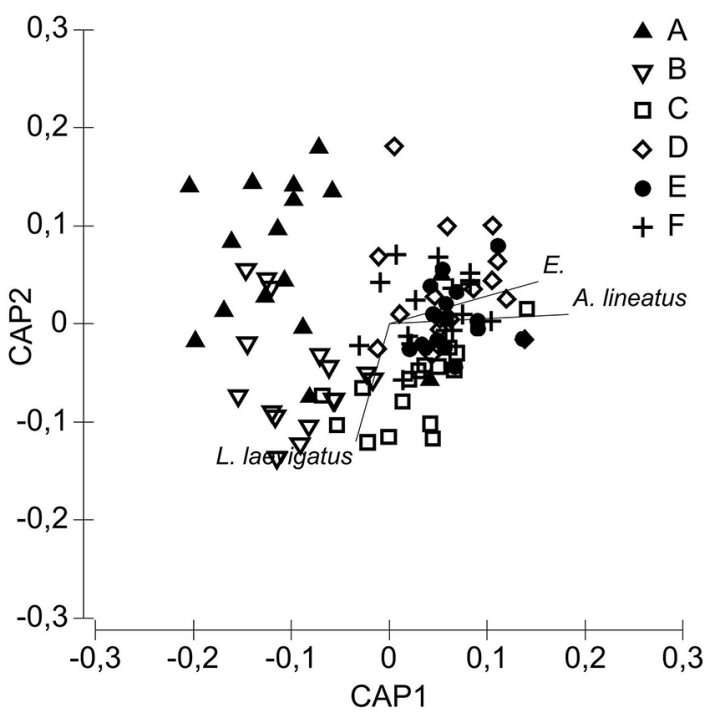

Figure 6. Result of the redundancy analysis based on the linear model (dbRDA) with the predictor variables showing the greatest importance for the linear model. Summer $=\mathrm{Su} ; \mathrm{Fall}=\mathrm{Fa}$; Winter $=\mathrm{Wi}$; Spring $=$ Sp. 
Regarding the indices of average taxonomic distinctness $($ Delta + ) and variation in taxonomic distinction (Lambda + ), associated with species richness, the PERANOVA indicated no significant differences for any of the variables and a similar taxonomic structure among seasons (Table 6). This is confirmed by the graphic result of the indices Lambda+ and Delta + , whose values were within the $95 \%$ confidence interval, calculated in 1000 simulations for each index (Figures 7a and 7b). Nevertheless, for Delta + , we observed that the fall, winter and summer samples were below the values expected (Figure 7a). For Lambda+, winter and summer samples were below the average (Figure $7 \mathrm{~b}$ ). The biplot graph of both indices showed that the Delta+ and Lambda+ of the spring were high (Figure 7c). For this last index, the value is greater than in summer and winter and lower than in fall. The location of the spring samples within the ellipse of $95 \%$ probability of occurrence of 60 species $(S=60)$ is a reflection of the high variation in taxonomic distinctness, despite having the highest average taxonomic distinctness, when compared with the other months. On the other hand, winter samples had a lower average taxonomic distinctness, i.e., a lower diversity in taxonomic structure; the variability is lower due to the low variance in the taxonomic distinction.

\section{DISCUSSION}

In North Bay, seasonality was pronounced, given the significant differences in salinity, temperature, $\mathrm{pH}$, and rainfall during the sampling year. Regarding rainfall index, an uncommon pattern was observed in 2005, with high levels of rainfall in winter. Despite this, the seasonal pattern expected for the area was maintained, since it is a temperate region with clear distinction between the warm seasons (spring and summer) and cold seasons (fall and winter). Spatially, the environmental parameters showed no significant variation. When evaluating their influence on fish abundance, by means of DistLM, it was observed that all parameters especially temperature and $\mathrm{pH}$ significantly influenced the abundance. Temperature is directly related to seasonality and has a direct effect on the metabolic and reproductive processes of fish fauna. In its turn, $\mathrm{pH}$ variation is more conditioned by the inflow of freshwater, mainly from mangrove areas, which are rich in humic and fulvic acids, responsible for the acidification of water (DAY; YAÑES-ARANCIBA, 1982; SIMONASSI et al., 2010). This pattern is consistent with the result of dbRDA, whose positive correlation of $\mathrm{pH}$ has been associated with springtime, a period with the lowest indices of rainfall for the year sampled and hence a lower inflow of freshwater into the system.

With respect to the structure of assemblages, the dominance of a few species of demersal fish has also been registered in other studies on the southeastern and southern coasts of Brazil (ROSSI-WONGTSCHOWSKI; PAES, 1993; ARAÚJO et al., 2002; HOSTIM-SILVA et al., 2002; SCHWARZ JR. et al., 2006; AZEVEDO et al., 2007; QUEIROZ et al., 2007; BARLETTA et al., 2008; PINHEIRO et al., 2009; CARTAGENA et al., 2011). The dominance of a few species seems to be an ecological pattern of demersal assemblages or an effect of the selectivity of the fishing gear. The common predominance of the families Ariidae and Sciaenidae was not observed in North Bay. Despite the high occurrence of these families, there were higher abundances of Paralichthyidae and Sciaenidae.

Seasonal differences in the abundance of the demersal fish fauna of the southeastern and southern regions are often observed (ROSSI-WONGTSCHOWSKI; PAES, 1993; HOSTIM-SILVA et al., 2002; ARAÚJO et al., 2002; AZEVEDO et al., 2007; QUEIROZ et al., 2007; BARLETTA et al., 2008; PINHEIRO et al., 2009; CARTAGENA et al., 2011), except for the fish fauna of the Pinheiros Bay, located in the Paranaguá estuarine complex (CEP) (SCHWARZ JR. et al., 2006). However, the lack of statistical seasonal differences in fish abundance in the Pinheiros Bay does not seem to be a pattern for CEP, in view of significant temporal differences on the main axis (east-west) of CEP (BARLETTA et al., 2008). Differences between the spatial scales adopted in these studies seem to have influenced seasonal patterns. In North Bay, high abundances were registered in the fall period and are probably a pattern for fish of the Southeastern and Southern coasts. The reproductive patterns, according to which the main species of the families Sciaenidae and Clupeidae spawn during the spring and summer, while recruiting in shallow areas, but which are still not part of demersal assemblages, might also explain such differences. In autumn and winter, recruited fish have reached maturity and occupy deeper areas of estuaries (PINHEIRO et al., 2009), increasing the probability of capture by trawl.

In the study area, fish species with relative abundance greater than $1 \%$, in descending order of importance, were $G$. genidens, C. spilopterus, C. chrysurus, $M$. furnieri and P. punctatus; the first three had relative 

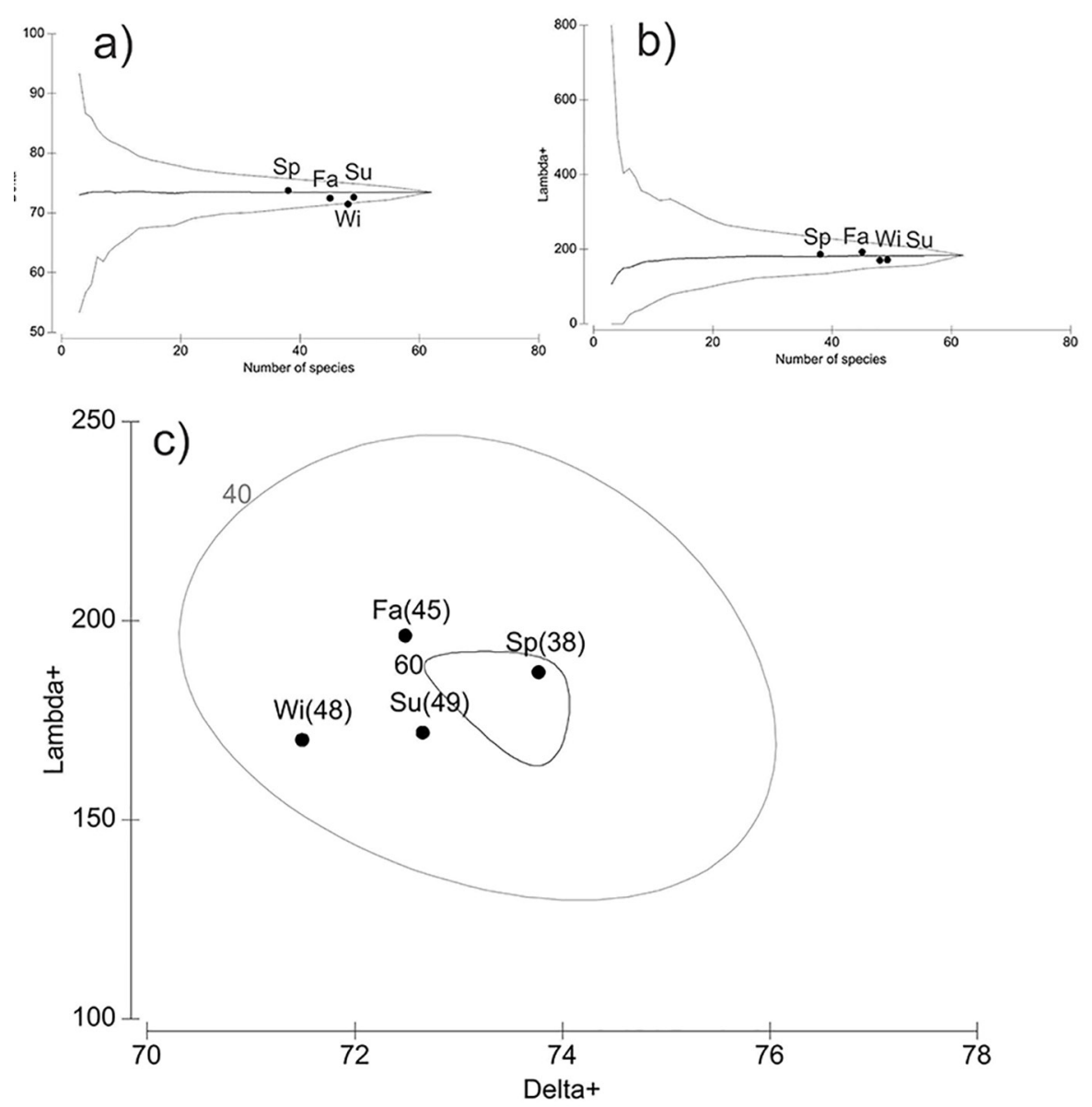

Figure 7. Average taxonomic distinctness (AvTD - DELTA + ) and variation in taxonomic distinction (VarTD - LAMBDA +) (b) calculated for the North Bay per season $($ Summer $=$ Su; Fall $=$ Fa; Winter $=$ Wi; Spring $=S p)$. For both indices, the expected average represented by the central dotted line and the $95 \%$ confidence interval limit is given by the surrounding funnel-shaped solid line. Biplot graphic display or LAMBDA+ and DELTA+ (c). The ellipse represents the value of the $95 \%$ confidence interval of the probability of finding 40 and 60 species, respectively.

abundance of approximately $40 \%$. On the southeastern and southern coasts of Brazil, G. genidens, C. chrysurus and P. puncatus are abundant (over 1\%) in demersal assemblages (AZEVEDO et al., 2007; QUEIROZ et al., 2007; BARLETTA et al., 2008. However, we registered high abundances of C. spilopterus in North Bay (the most abundant species in this area) and a low occurrence of $S$. rastrifer, which is not consistent with these fish surveys. Although C. spilopterus is not commonly recorded in the demersal fish fauna of the southeastern and southern coasts of Brazil, it is a shallow-water species, living especially in estuarine areas (FIGUEREDO; MENEZES, 2000; FROSE; PAULY, 2014).

The highest abundance of $C$. spilopterus was found in fall and winter in North Bay. In Guaratuba Bay, this was observed in the spring and summer, with spawning occurring in mangrove areas, in late spring and early summer (CHAVES; VENDEL, 1997). The high catch of C. spilopterus may be associated with the presence of the mangroves of Itacorubi and Ratones on the bay's shores. This is confirmed by the highest occurrences of this species in areas $\mathrm{C}$ and $\mathrm{E}$, which are close to these mangroves.

The high abundance of $G$. genidens is expected in the demersal assemblages of the southern and southeastern coasts of Brazil (HOSTIM-SILVA et al., 2002; QUEIROZ et al., 2007; BARLETTA et al., 2008; CARTAGENA et al., 2011). In a comparison of the fish fauna of five estuaries along the Brazilian coast, there was a predominance of this species on the southern and southeastern coasts (VILAR et al., 2013). In North Bay, high abundances 
were registered in all four seasons, mainly in summer and spring. Higher abundances of $G$. genidens occurred in the headwaters of the east-west axis of the Paranaguá Estuarine Complex, in fall and winter (BARLETTA et al., 2008). The numerous presence of $G$. genidens in shallow estuarine areas was also verified in Sepetiba Bay (AZEVEDO et al., 1999; 2007). It is a predominantly estuarine species, inhabiting areas near rivers and displaying the ability to withstand high variations in salinity (FIGUEREDO; MENEZES, 1978; FROESE; PAULY, 2014). The highest abundances occurred in areas B, C and E, associated with the rivers Biguaçú (area B), Ratones (C, E) and Itacorubi (E). As for the sizes, higher values of total length (TL) were observed in fall and winter, which might coincide with the species' reproductive activity.

C. chrysurus is another species with high abundances in North Bay. It is a pelagic species occurring in estuaries and bays (MENEZES; FIGUEREDO, 1980; FROESE; PAULY, 2014). High abundances were also reported in the Paranaguá Estuarine Complex (BARLETTA et al., 2008 ) and in inner regions of Sepetiba Bay in spring and summer (ARAÚJO et al., 2002; AZEVEDO et al., 2007). The greatest abundance occurred in the winter in area B. Although differences in abundance between seasons are distinct from those given by other studies, depths were shallow in both areas. The high incidence of pelagic species in shallow areas of Pinheiros Bay (SCHWARZ JR. et al., 2006) has already been observed. Thus, the high prevalence of this species may be related to the shallow depth of the sampled sites.

It may thus be concluded that seasonality has a structuring effect on the fish assemblage in North Bay. However, it remained unclear which is the main regulatory mechanism of assemblages. Although seasonal differences are directly reflected in the physical and chemical parameters of the water column, which, in turn, influence the patterns of distribution and occurrence of demersal assemblages (BLABER; BLABER, 1980; MARSHALL; ELLIOT, 1998; BLABER, 2000; WHITFIELD et al., 2012), in North Bay, environmental gradients were not well-delimited, as is usually observed in estuarine areas. Possibly the ecological processes of reproduction, spawning and recruitment have greater influence on the patterns of dominance, given the high abundance of smaller sized individuals of a few species. The results presented here highlight the importance of North Bay as a nursery of fish of commercial importance and others that may potentially feed the fish stocks of the network of marine protected areas of the state of Santa Catarina.

\section{ACKNOWLEDGMENTS}

We wish to thank the fisher Lourenço for his help in data collection, as well as many other people for their help in the field and the laboratory, specially Carlos Espírito Santos. FAPESC (Fundação de Amparo à Pesquisa e Inovação do Estado de Santa Catarina) provided financial support for the data collection. CNPq provided a master's scholarship for FDG and LLW and CAPES provided a PhD scholarship for APC.

\section{REFERENCES}

ANDERSON M. J.; GORLEY R. N.; CLARKE K. R. PERMANOVA + for PRIMER: guide to software and statistical methods. Plymouth: PRIMER-E, 2008.

ARAÚJO, F. G.; AZEVEDO, M. C. C.; PESSANHA, A. L. M.; SILVA, M. A.; GOMES, I. D.; CRUZ-FILHO, A. G. Environmental influences on the demersal fish assemblages in the Sepetiba Bay, Brazil. Estuaries, v. 25, n. 3, p. 441-450, 2002.

AZEVEDO, M. C. C; ARAÚJO, F. G.; CRUZ-FILHO, A. G.; GOMES, I. D.; PESSANHA, A. L. M. Variação espacial e temporal de bagres marinhos (Silurifomres, Ariidae) na Baía de Sepetiba, Rio de Janeiro. Rev. Bras. Biol., v. 59, n. 3, p. 443-454, 1999.

AZEVEDO, M. C. C.; ARAÚJO, F. G.; CRUZ-FILHO, A. G.; PESSANHA, A. L. M.; SILVA, M. A.; GUEDES, A. P. P. Demersal fishes in a tropical bay in southeastern Brazil: Partitioning the spatial, temporal and environmental components of ecological variation. Estuar. Coast. Shelf Sci., v. 75, n. 4, p. 468-480, 2007.

BARLETTA, M.; AMARAL, C. S.; CORRÊA, M. F. M.; GUEBERT, F.; DANTAS, D. V.; LORENZI, L.; SAINTPAUL, U. Factors affecting seasonal variations in demersal fish assemblages at an ecocline in a tropical-subtropical estuary. J. Fish. Biol., v. 73, n. 6, p. 1314-1336, 2008.

BARLETTA, M.; BARLETTA-BERGAN, A.; SAINT-PAUL, U.; HUBOLD, G. The role of salinity in structuring the fish assemblages in a tropical estuary. J. Fish. Biol., v. 66, n. 1, p. 45-72, 2005.

BARREIROS, J. P.; BRANCO, J. O.; FREITAS JUNIOR, F; MACHADO, L. F.; HOSTIM-SILVA, M.; VERANI, J. R. Space-time distribution of the ichthyofauna from Saco da Fazenda Estuary, Itajaí, Santa Catarina, Brazil. J. Coastal. Res., v. 25, p. 1114-1121, 2009.

BLABER, S. J. M. Tropical estuarine fishes: ecology, exploitation and conservation. Oxford: Blackwell Science, 2000. 372 p.

BLABER, S. J. M.; BLABER, T. G. Factors affecting the distribution of juvenile estuarine and inshore Fish. J. Fish. Biol., v. 17, n. 2, p. 143-162, 1980.

BREHMER, P.; LAUGIER, T.; KANTOUSSAN, J.; GALGANI, F.; MOUILLOT, D. Does coastal lagoon habitat quality affect fish growth rate and their recruitment? Insights from fishing and acoustic surveys. Estuar. Coast. Shelf Sci., v. 126, p. 1-6, 2013.

BURNS, M. D. M.; GARCIA, A. M.; VIEIRA, J. P.; BENVENUTI, M. A.; MARQUES, D. M. L. M.; CONDINI, $\mathrm{V}$. Evidence of habitat fragmentation affecting fish movement 
between the Patos and Mirim coastal lagoons in southern Brazil. Neotrop. Ichthyol., v. 4, n. 1, p. 69-72, 2006.

CABRAL, H. N.; COSTA, M. J.; SAlGADO, J. P. Does the Targus estuary fish community reflect environmental changes? Clim. Res., v. 18, p. 119-126, 2001.

CARTAGENA, B. F. C.; HOSTIM-SILVA, M.; SPACH, H. L. Distribuição espacial da ictiofauna demersal no Saco dos Limões (Baía Sul, Florianópolis, SC). Bioikos, v. 25, n. 2, p. 117-128, 2011

CHAVES, P. T. C.; VENDEL, A. L. Indicadores reprodutivos das espécies de Citharichthys Bleeker (Teleostei, Pleuronectiformes) na Baia de Guaratuba, Paraná, Brasil. Rev. Bras. Zool., v. 14, n. 1, p. 73-79, 1997.

CLARKE, K. R.; WARWICK, R. W. Change in marine communities: an aproach to statistical analysis and interpretation. Plymouth: Plymouth Marine Laboratory, 1994. $144 \mathrm{p}$.

DAY JR, J. W.; YÁÑEZ-ARANCIBIA, A. Coastal lagoon and estuarines: ecosystem approach. Ciênc. Interam., v. 22, n. 1-2, p. 11-26, 1982.

ELLIOTT, M.; QUINTINO, V. The Estuarine Quality Paradox, Environmental Homeostasis and the difficulty of detecting anthropogenic stress in naturally stressed areas. Mar. Pollut. Bull., v. 54, n. 6, p. 640-645, 2007.

ENTE, R. F.; STEFANONI, M. F.; SPACH, H. L. Feeding ecology of the Brazilian silverside Atherinella brasiliensis (Atherinopsidae) in a sub-tropical estuarine ecosystem. J. Mar. Biol. Assoc. U. K., v. 91, n. 6, p. 1197-1205, 2011.

FALCÃO, M. G.; SARPÉDONTI, V.; SPACH, H. L.; OTERO, M. E. B.; QUEIROZ, G. M. L. N.; SANTOS, C. A ictiofauna em planícies de maré das Baías de Laranjeiras e de Paranaguá, Paraná, Brasil. Rev. Bras. Zoocienc., v. 8, n. 2, p. $125-138,2006$.

FIGUEIREDO, J. L.; MENEZES, N. A. Manual de Peixes Marinhos do Sudeste do Brasil. II. Teleostei (1). São Paulo: Museu de Zoologia da Universidade de São Paulo, 1978, $110 \mathrm{p}$.

FIGUEIREDO, J. L.; MENEZES, N. A. Manual de peixes marihos do sudeste do Brasil. VI. Teleostei (5). São Paulo: Museu de Zoologia da Universidade de São Paulo, 2000. 116 p.

FROESE, R. D.; PAULY, D. FishBase. World Wide Web electronic publication. Available: < http://www.fishbase.org/ home.htm>. Accessed: 15 Oct. 2014.

GARCIA, A. M.; RASEIRA, M. B.; VIEIRA, J. P.; WINEMILLER, K. O.; GRIMM, A. M. Spatiotemporal variation in shallow-water freshwater fish distribution and abundance in a large subtropical coastal lagoon. Environ. Biol. Fishes., v. 68, n. 3, p. 215-228, 2003.

GARCIA, A. M.; VIEIRA, J. P. O aumento da diversidade de peixes no estuário da Lagoa dos Patos durante o episódio El Niño 1997-1998. Atlântica, v. 23, p. 133-152, 2001a.

GARCIA, A. M.; VIEIRA, J. P.; WINEMILLER, K. O. Dynamics of the shallow-water fish assemblage of the Patos Lagoon estuary (Brazil) during cold and warm ENSO episodes. J. Fish. Biol., v. 59, n. 5, p. 1218-1238, 2001 b.

GREENWOOD, M. F. D.; HILL, A. S. Temporal, spatial and tidal influences on benthic and demersal fish abundance in the Forth estuary. Estuar. Coast. Shelf Sci., v. 58, n. 2, p. 211-225, 2003.
HARRISON, T. D.; WHITFIELD, A. K. Temperature and salinity as primary determinants influencing the biogeography of fishes in South Africa estuaries. Estuar Coast. Shelf Sci., v. 66, n. 1-2, p. 335-345, 2006.

HOSTIM-SILVA, M.; VICENTE, M. J. D.; FIGNA, V.; ANDRADE, J. P. Ictiofauna do rio Itajaí Açu, Santa Catarina, Brasil. Notas Téc. Facimar, v. 6, p. 127-135, 2002.

JAUREGUIZAR, A. J.; MENNI, R.; GUERREIRO, R.; LASTA, C. Environmental factors structuring fish communities of the Rio de la Plata estuary. Fish. Res., v. 66, n. 2/3, p. 195-211, 2004.

KENNISH, M. J. Ecology of estuaries. Boca Raton: CRC Press, 1990.

LOBRY, J.; MOURAND, L.; ROCHARD, E.; ELIE, P. Structure of the Gironge estuarine fish assemblages: a comparison of European estuaries perspective. Aquat. Living Resour., v. 16, n. 2, p. 47-58, 2003.

MACI, S.; BASSET, A. Composition, structural characteristics and temporal patterns of fish assemblages in non-tidal Mediterranean lagoons: A case study. Estuar. Coast. Shelf Sci., v. 83, n. 4, p. 602-612, 2009.

MARSHALL, S.; ELLIOTT, M. Environmental Influences on the Fish Assemblage of the Humber Estuary, U.K. Estuar. Coast. Shelf Sci., v. 46, n. 2, p. 175-184, 1998.

MC DOWALL, R. M. Diadromy in fishes, migrations between freshwater and marine environments. Portland: Timber Press, 1988.

MENEZES, N. A.; FIGUEIREDO, J. L. Manual de peixes marinhos do sudeste do Brasil. IV. Teleostei (3). São Paulo: Museu de Zoologia da Universidade de São Paulo, 1980. 96 p.

MONTEIRO-NETO, C.; BLACHER, C.; LAURENT, A. A. S.; SNIZEK, F. N.; CANOZZI, M. B.; TABAJARA, L. L. C. Estrutura da comunidade de peixes de águas rasas na região de Laguna, Santa Catarina, Brasil. Atlântica, v. 12 , n. 2, p. 53-69, 1990.

OTERO, M. E. B.; SPACH, H. L.; PICHLER, H. A.; QUEIROZ, G. M. N.; SANTOS, C.; SILVA, A. L. C. O uso de atributos das assembleias de peixes para avaliar a integridade biótica em habitats rasos das Baías de Antonina e Paranaguá, Paraná. Acta Biol. Par., v. 35, n. 1-2, p. 69-82, 2006.

PINHEIRO, H. T.; MARTINS, A. S.; ARAÚJO, J. N.; PINTO, A. S. S. Evidence of seasonal changes in community structure for a coastal ecosystem in the central coast of Brazil, southwest Atlantic. J. Mar. Biol. Ass. U. K., v. 89, n. 2, p. 217-224, 2009.

QUEIROZ, G. M. L. N., SPACH, H. L., SOBOLEWSKI-MORELOS, M.; SCHWARZ JUNIOR, R. A ictiofauna demersal de áreas com diferentes níveis de ocupação humana, no estuário de Paranaguá. Arq. Cienc. Mar., v. 40, n. 2, p. 80-91, 2007.

RAMOS, L. A.; VIEIRA, J. P. Composição específica e abundância de peixes de zonas rasas dos cinco estuários do Rio Grande do Sul, Brasil. Bol. Inst. Pesca, v. 27, n. 1, p. 109-121, 2001.

RAZ-GUZMAN, A.; HUIDOBRO, L. Fish communities in two environmentally different estuarine systems of Mexico. J. Fish Biol., v. 60, p. 1-14, 2002.

RIBEIRO, G. C.; CLEZAR, L.; HOSTIM-SILVA, M.; FILOMENO, M. J. B.; AGUIAR, J. B. S. Ictiofauna da lagoa 
da Conceição e área costeira adjacente, Ilha de Santa Catarina, SC, Brasil. Aquitaine Océan, v. 1, n. 3, p. 270-281, 1997.

RIBEIRO, G. C.; CLEZAR, L.; SILVA, M. H. Comunidade ictíica, sua variação espacial e sazonal na Lagoa da Conceição e área costeira, Ilha de Santa Catarina, SC, Brasil: o ecossistema da Lagoa da Conceição. Ser. Fepema, v. 4, p. 261-273, 1999.

RIBEIRO, G. C.; SOETH, M.; ANDRADE, V. K.; SPACH, H. L.; CATTANI, A. P. Nycthemeral and Monthly Occupation of the Fish Assemblage on a Sheltered Beach of Baía Norte, Florianópolis, Santa Catarina State, Brazil. Braz. J. Oceanogr., v. 62, n. 3, p. 209-223, 2014

ROSSI-WONGTSCHOWSKI, C. L. D. B.; PAES, E. T. Padrões espaciais e temporais da comunidade de peixes demersais do litoral norte do Estado de São Paulo, Ubatuba, Brasil. Publ. Esp. Inst. Oceanogr., v. 10, p. 169-188, 1993.

SCHWARZ JR, R.; FRANCO, A. C. N. P.; SPACH, H. L.; SARPEDONTI, V.; PICHLER, H. A.; QUEIROZ, G. M. N. L. Composição e estrutura da ictiofauna demersal na Baía dos PInheiros, Paraná. Braz. J. Aquat. Sci. Technol., v. 10, n. 1, p. 27-39, 2006.

SHEAVES, M.; JOHNSTON, R. Ecological drivers of spatial variability among fish fauna of 21 tropical Australian estuaries. Mar. Ecol. Prog. Ser., v. 385, p. 245-260, 2009.

SIMONASSI, J. C.; HENNEMANN, M. C. TALGATTI, D.; MARQUES JR, A. N. Nutrient variations and coastal water quality of Santa Catarina Island, Brazil. Biotemas, v. 23, n. 1, p. 211-223, 2010.

SORIANO-SIERRA, E. J. O Ecossistema da Lagoa da Conceição. Florianópolis: Fepema, 1999. p. 25-34.
SORIANO-SIERRA, E. J.; SIERRA DE LEDO, B. Ecologia e gerenciamento do manguezal de Itacorubí. Florianópolis: NEMAR, 1998. $396 \mathrm{p}$.

SPACH, H. L.; FÉLIX, F. C.; HACKRADT, C. W.; LAUFER, D. C.; MORO, P. S.; CATTANI, A. P. Utilização de ambientes rasos por peixes na Baía de Antonina, Paraná. Biociências, v. 14, n. 2, p. 125-135, 2006.

SPACH, H. L.; GODEFROID, R. S.; SANTOS, C.; SCHWARZ JR, R.; QUEIROZ, G. M. L. Temporal variation in fish assemblage composition on a tidal flat. Braz. J. Oceanogr., v. 52, n. 1, p. 47-58, 2004.

SPACH, H. L.; SANTOS, C.; PICHLER, H. A.; IGNÁCIO, J. M.; STOIEV, S. B.; BERNARDO, C. Padrões estruturais da assembleia de peixes em duas áreas do Canal da Cotinga, Baía de Paranaguá, Paraná, Brasil. Bioikos, v. 21, n. 2, p. 57-67, 2007.

VILAR, C. C.; JOYEUX, J. C.; GIARRIZZO, T.; SPACH, H. L.; VIEIRA, J. P.; VASKE-JUNIOR, T. Local and regional ecological drivers of fish assemblages in Brazilian estuaries. Mar. Ecol. Prog. Ser., v. 485, p. 181-197, 2013.

VILAR, C. C.; SPACH, H. L.; JOYEUX, J. C. Spatial and temporal changes in the fish assemblage of a subtropical estuary in Brazil: environmental effects. J. Mar. Biol. Assoc. U. K., v. 91, n. 3, p. 635-648, 2011.

WHITFIELD, A. K.; ELLIOTT, M.; BASSET, A.; BLABER, S. J. M.; WEST, R. J. Paradigms in estuarine ecology - A review of the Remane diagram with a suggested revised model for estuaries. Estuar. Coast. Shelf Sci., v. 97, p. 78-90, 2012. 